\title{
Synthesis and characterization of new aromatic esters based on 4,16-pregnadiene-6,20-dione skeleton
}

\author{
Juan Li, Hongqi Li*, Yijing Li
}

\begin{abstract}
A series of new aromatic esters based on 4,16-pregnadiene-6,20-dione skeleton, namely 3ß-benzoyloxy-4,16-pregnadiene-6,20-dione and 33-furoyloxy-4,16-pregnadiene-6,20-dione, which may be good inhibitors for the $5 \alpha$ reductase enzyme and show high antiandrogenic activity, were synthesized starting from diosgenin. The structures of the steroids were characterized by elemental analysis, ${ }^{1} \mathrm{H} N \mathrm{NMR},{ }^{13} \mathrm{C} N \mathrm{NR}$, IR and mass spectrum. Single crystal $\mathrm{X}$ ray diffraction measurement on one of the new compounds, 33-( $p$-methoxybenzoyloxy)-4,16-pregnadiene-6,20dione revealed that the $A, B, C$, and D ring adopted half chair, distorted chair, distorted chair, and distorted envelope conformation, respectively. The molecules in the crystal were packed face-to-face at the normal van der Waals distances.
\end{abstract}

\section{Introduction}

Studies on steroids stimulated renewed interest because apart from their estrogenic and anabolic/androgenic activity [1-3] steroids were found to show various biological activities including cytotoxicity [4], antiproliferative activity [5], antimicrobial activity [6], anti-glioma activity [7], inhibition of cholesterol $\alpha$-glucosyltransferase [8], neuromuscular blocking activity [9], differential activities on prostate cancer cells [10], anti-osteoporosis activity [11], and anti-aging activity [12]. Up until now steroids have showed potential applications as $5 \alpha$-reductase inhibitors [13], as modulators of inflammation and immunity [14], as inhibitors of $17 \alpha$-hydroxylase $/ C_{17,20}$-lyase [15], as predictor of tamoxifen response in premenopausal breast cancer [16], as progesterone receptor antagonist [17], as glucocorticoid receptor imaging agents [18], as inhibitors of protein tyrosine phosphatase 1B [19], as reversal agents of multidrug resistance in cancer cells [20], as inhibitors of $17 \beta$-hydroxysteroid dehydrogenase [21], and as ligands for drug vectors [22]. Some steroidal compounds were used for photodynamic therapy [23], for cancer chemotherapy [24], and for DNA delivery [25]. Moreover, cholesteric liquid crystal [26] and

\footnotetext{
* Correspondence: hongqili@dhu.edu.cn Key Laboratory of Science \& Technology of Eco-Textile, Ministry of Education, College of Chemistry, Chemical Engineering \& Biotechnology, Donghua University, 2999 North Renmin Road, Shanghai 201620, P. R. China
}

steroid-based organogelator [27] were also studied. Recently Cabeza and collegues synthesized a series of $3 \beta$-benzoyloxy-4,16-pregnadiene-6,20-dione (1a-e, Figure 1 ) and $3 \beta$ - cycloalkylcarbonyloxy-4,16-pregnadiene-6,20-dione (2a-d, Figure 1) compounds starting from 16-dehydropregnenolone acetate (16-DPA) and evaluated their biological activities. Of these steroids 1a is a good inhibitor for the $5 \alpha$-reductase enzyme [13] while $\mathbf{1 b}$-e and 2a-d showed high antiandrogenic activity $[28,29]$. In continuation of our study on steroids $[30,31]$ and to explore an improved way for synthesis of new steroidal compounds from the cheap starting material diosgenin, herein we report the synthesis and structural characterization of a series of new aromatic esters based on 4,16-pregnadiene-6,20-dione skeleton.

\section{Experimental}

All chemical reagents were purchased from commercial sources and used as received unless stated otherwise. Melting points were determined on a XT-4 melting apparatus and the quoted temperatures were uncorrected. Elemental analysis was carried out on an Elmentar Vario EL III system. ${ }^{1} \mathrm{H}$ NMR and ${ }^{13} \mathrm{C}$ NMR spectra were recorded on a Bruker AM 400 spectrometer. $\mathrm{CDCl}_{3}$ was used as solvent and chemical shifts recorded were internally referenced to $\mathrm{Me}_{4} \mathrm{Si}(0 \mathrm{ppm})$. IR spectra were obtained on a Thermo Electron Corporation 
<smiles>[R]c1ccc(C(=O)O[C@H]2C=C3C(=O)C[C@H]4[C@@H]5CC=C(C(C)=O)[C@@]5(C)CC[C@H]4[C@@]3(C)CC2)cc1</smiles>

1a: $\mathrm{R}=\mathrm{H}$

1b: $R=F$

1c: $\mathrm{R}=\mathrm{Br}$

1d: $\mathrm{R}=\mathrm{Cl}$

1e: $\mathrm{R}=\mathrm{Me}$<smiles>[R]C(=O)O[C@H]1C=C2C(=O)C[C@H]3[C@@H]4CC=C(C(C)=O)[C@@]4(C)CC[C@H]3[C@@]2(C)CC1</smiles>

2a: $\mathrm{R}=$<smiles>CC1CC1</smiles>

2b: $\mathrm{R}=$<smiles>CC1CCC1</smiles>

2c: $\mathrm{R}=$<smiles>CC1CCCC1</smiles>

2d: $\mathrm{R}=$

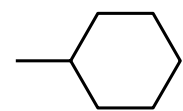

Figure 1 Chemical structure of compound 1a-e and 2a-d

Nicolet 380 FT-IR spectrophotometer. Mass spectra were recorded on a Shimadzu QP-2010 instrument using electron impact ionization (EI) at $70 \mathrm{eV}$. LC-MS spectra were obtained on an Agilent 6000 LC-MS instrument equipped with a SunFire C18 column $(4.6 \times$ $50 \mathrm{~mm}, 3.5 \mu \mathrm{m}$ ) under the following conditions: mobile phase: $\mathrm{H}_{2} \mathrm{O}(0.05 \%$ trifluoroacetic acid (TFA)) (A)/acetonitrile (0.05\% TFA) (B); elution program: gradient from 5 to $95 \%$ of B in $1.6 \mathrm{~min}$ at $2.2 \mathrm{ml} / \mathrm{min}$; temperature: $50^{\circ}$; detection: UV (214 $\mathrm{nm}$ ) and MS (ESI, pos mode, 70 to $1000 \mathrm{amu}$ ). All of the measured samples were dissolved in methanol. X-Ray crystal structure was measured on a Bruker Smart CCD diffractometer by using Mo $\mathrm{K}_{\alpha}$ radiation at $293 \mathrm{~K}$.

\section{Pseudodiosgenin diacetate (4) [32]}

To a solution of diosgenin $3(16.0 \mathrm{~g}, 38.6 \mathrm{mmol})$ and ammonium chloride $(2.5 \mathrm{~g}, 46.7 \mathrm{mmol})$ in acetic anhydride $(82 \mathrm{ml})$ was added pyridine $(1.5 \mathrm{ml})$ and the mixture was heated at $140^{\circ} \mathrm{C}$ for $8-9 \mathrm{~h}$. The resulting solution was cooled to room temperature and poured into ice water. The precipitate was filtered and recrystallized from methanol to give the product pseudodiosgenin diacetate (4) as yellow solid (16.6 g, 86\%). M.p. $70-72^{\circ} \mathrm{C}$.

\section{$3 \beta$-Acetoxy-5,16-pregnadiene-20-one (5)}

A solution of pseudodiosgenin diacetate (4) (15.4 g, 30.8 $\mathrm{mmol})$ in acetic anhydride $(76 \mathrm{ml})$ was diluted with water $(4.8 \mathrm{ml})$ and acetic acid $(50 \mathrm{ml})$. The mixture was cooled to $0^{\circ} \mathrm{C}$ then a solution of chromium(VI) oxide
$(8.9 \mathrm{~g}, 89.0 \mathrm{mmol})$ in acetic acid $(25 \mathrm{ml})$ was added dropwise in $1 \mathrm{~h}$. After the addition the solution was allowed to warm to $10-16^{\circ} \mathrm{C}$ and the stirring was continued for $5 \mathrm{~h}$ at this temperature. Sodium bisulphite $(9.3$ $\mathrm{g}, 89.4 \mathrm{mmol})$ in water $(30 \mathrm{ml})$ was added then the mixture was refluxed for $3 \mathrm{~h}$, cooled and poured into water to give sticky solids. The crude product was crystallized from methanol then purified by means of column chromatography on silica gel with petroleum ether (b.p. 60$\left.90^{\circ} \mathrm{C}\right)$ and ethyl acetate $(4: 1, \mathrm{v} / \mathrm{v})$ as eluent to afford product 5 (4.5 g, $41 \%)$ as yellow needles. M.p. $170-172^{\circ} \mathrm{C}$ (literature [32] value: $171-172^{\circ} \mathrm{C}$ ). $\mathrm{R}_{\mathrm{f}} 0.58$ (petroleum ether/ethyl acetate, $4: 1, \mathrm{v} / \mathrm{v})$.

Compound 6 was synthesized as described in literature [33] in 90\% yield and as described in literature [13] in $95 \%$ yield. M.p. $170-172^{\circ} \mathrm{C}$ (literature [13] value: $170-$ $172^{\circ} \mathrm{C}$ ). $R_{\mathrm{f}} 0.57$ (petroleum ether/ethyl acetate, 4:1, v/v).

Compound 7 was synthesized as described in literature [13] in $84 \%$ yield. M.p. $243-245^{\circ} \mathrm{C}$ (literature [13] value: $244-245^{\circ} \mathrm{C}$ ). $\mathrm{R}_{\mathrm{f}} 0.56$ (petroleum ether/ethyl acetate, $4: 1, \mathrm{v} / \mathrm{v})$.

Compound 8 was synthesized as described in literature [13] in $73 \%$ yield. M.p. $192-194^{\circ} \mathrm{C}$ (literature [13] value: $193-195^{\circ} \mathrm{C}$ ). $\mathrm{R}_{\mathrm{f}} 0.58$ (petroleum ether/ethyl acetate, $4: 1, \mathrm{v} / \mathrm{v})$.

\section{3ß-Hydroxypregna-4, 16-diene-6,20-dione (9)}

A solution of steroid $8(0.3 \mathrm{~g}, 0.8 \mathrm{mmol})$ in methanol $(45 \mathrm{ml})$ and $2 \%$ aqueous sodium hydroxide $(3 \mathrm{ml})$ was stirred for $30 \mathrm{~min}$ at room temperature. The resulting solution was poured into ice water $(45 \mathrm{ml})$ then 
extracted with ethyl acetate $(3 \times 30 \mathrm{ml})$. The organic phase was dried over anhydrous magnesium sulfate and the solvent was removed in vacuum. The residue was purified by column chromatography on silica gel with petroleum ether and ethyl acetate $(1: 1, \mathrm{v} / \mathrm{v})$ as eluent to afford product $9(0.25 \mathrm{~g}, 74 \%)$ as pale yellow needles. $\mathrm{M}$. p. $168-170^{\circ} \mathrm{C}$ (literature [13] value: $168-170^{\circ} \mathrm{C}$ ). $\mathrm{R}_{\mathrm{f}} 0.52$ (petroleum ether/ethyl acetate, 4:1, v/v).

\section{3 $\beta$-( $p$-Methoxybenzoyloxy)pregna-4,16-diene-6,20-dione (10a)}

A solution containing steroid $9(0.10 \mathrm{~g}, 0.3 \mathrm{mmol}), p$ methoxybenzoic acid $(0.13 \mathrm{~g}, 0.7 \mathrm{mmol}), 1,3$-dicyclohexylcarbodiimide (DCC, $0.10 \mathrm{~g}, 0.5 \mathrm{mmol}$ ) and 4-dimethylaminopyridine (DMAP, $0.06 \mathrm{~g}, 0.5 \mathrm{mmol}$ ) in methylene dichloride $(6 \mathrm{ml})$ was stirred for $2 \mathrm{~h}$ at about $10^{\circ} \mathrm{C}$. Ice water was added and the reaction mixture was extracted with chloroform $(3 \times 20 \mathrm{ml})$. The organic phase was dried over anhydrous magnesium sulfate and the solvent was removed in vacuum. The crude product was dissolved in ethyl acetate and filtered through a column containing silica gel. The organic solvent was removed in vacuum to give a white crystalline solid 10a $(0.14 \mathrm{~g}$, $71 \%$ ). M.p. $244-246^{\circ} \mathrm{C} . \mathrm{R}_{\mathrm{f}} 0.50$ (petroleum ether/ethyl acetate, 4:1, v/v). ${ }^{1} \mathrm{H}$ NMR $\left(\mathrm{CDCl}_{3}\right): 0.94(\mathrm{~s}, 3 \mathrm{H}, \mathrm{H}-\mathrm{C}$ (18)); 1.10 (s, 3 H, H-C(19)); 2.28 (s, 3 H, H-C(21)); 3.87 (s, $3 \mathrm{H}, \mathrm{OMe}) ; 5.56$ (m, $1 \mathrm{H}, \mathrm{H}-\mathrm{C}(3)) ; 6.23(\mathrm{~m}, 1 \mathrm{H}, \mathrm{H}-\mathrm{C}$ (4)); $6.71(\mathrm{t}, 1 \mathrm{H}, J=1.3 \mathrm{~Hz}, \mathrm{H}-\mathrm{C}(16)) ; 6.93(\mathrm{~m}, 2 \mathrm{H}, \mathrm{H}-$ $\mathrm{Ph}) ; 8.02(\mathrm{~m}, 2 \mathrm{H}, \mathrm{H}-\mathrm{Ph}) .{ }^{13} \mathrm{C}$ NMR $\left(\mathrm{CDCl}_{3}\right): 15.8$ (C (18)); 19.7 (C(19)); 27.1 (C(21)); 69.5 (C(3)); 131.8 (C (4)); 143.8 (C(16)); 147.9 (C(5)); 154.9 (C(17)); 165.9 (ester $\mathrm{C}=\mathrm{O})$; $196.6(\mathrm{C}(20))$; $201.9(\mathrm{C}(6))$. IR ( $\mathrm{KBr})$ : 3075, 2958, 1706, 1690, 1656, 1629. EI-MS: 312 (12, $\mathrm{M}^{+}$ + $1-p-\mathrm{MeO}-\mathrm{C}_{6} \mathrm{H}_{4} \mathrm{COO}$ ), 175 (31), 157 (38), 121 (30), 105 (41), 93 (33), 91 (66), 79 (51), 77 (46), 43 (100). LCMS: 485.4 $\left(\mathrm{M}^{+}+\mathrm{Na}\right.$, retention time $\left.2.20 \mathrm{~min}\right)$. Anal. Calcd. for $\mathrm{C}_{29} \mathrm{H}_{34} \mathrm{O}_{5}$ : C, 75.30; H, 7.41; Found: C, 75.12; $\mathrm{H}, 7.37$.

Other steroidal compounds $\mathbf{1 0 b}-\mathbf{j}$ were prepared in a similar procedure and the physical data of the new steroids were as follows.

\section{3ß-(o-Methoxybenzoyloxy)pregna-4,16-diene-6,20-dione (10b)}

White powder. Yield $45 \%$. M.p. $240-242^{\circ} \mathrm{C}$. $\mathrm{R}_{\mathrm{f}} 0.42$ (petroleum ether/ethyl acetate, 4:1, v/v). ${ }^{1} \mathrm{H}$ NMR $\left(\mathrm{CDCl}_{3}\right)$ : 0.93 (s, 3 H, H-C(18)); 1.07 (s, 3 H, H-C(19)); 2.27 (s, 3 $\mathrm{H}, \mathrm{H}-\mathrm{C}(21))$; 3.89 (s, $3 \mathrm{H}, \mathrm{OMe})$; 5.56 (m, $1 \mathrm{H}, \mathrm{H}-\mathrm{C}(3)$ ); $6.23(\mathrm{~m}, 1 \mathrm{H}, \mathrm{H}-\mathrm{C}(4))$; $6.73(\mathrm{t}, J=1.6 \mathrm{~Hz}, \mathrm{H}-\mathrm{C}(16)) ; 6.96$ $(\mathrm{m}, 2 \mathrm{H}, \mathrm{H}-\mathrm{Ph}) ; 7.46(\mathrm{~m}, 1 \mathrm{H}, \mathrm{H}-\mathrm{Ph}) ; 7.80(\mathrm{~m}, 1 \mathrm{H}, \mathrm{H}-$ Ph). ${ }^{13} \mathrm{C}$ NMR $\left(\mathrm{CDCl}_{3}\right): 17.5$ (C(18)); 22.0 (C(19)); 26.5 (C(21)); $71.8(\mathrm{C}(3)) ; 136.0(\mathrm{C}(4)$ and $\mathrm{C}(16)) ; 146.1(\mathrm{C}(5))$; $149.9(\mathrm{C}(17)) ; 167.0$ (ester $\mathrm{C}=\mathrm{O})$; $198.9(\mathrm{C}(20)) ; 203.8$ (C(6)). IR (KBr): 3040, 2946, 1688. LC-MS: 485.4 $\left(\mathrm{M}^{+}+\right.$
$\mathrm{Na}$, retention time $2.13 \mathrm{~min})$. Anal. Calcd. for $\mathrm{C}_{29} \mathrm{H}_{34} \mathrm{O}_{5}$ : C, 75.30; H, 7.41; Found: C, 75.04; H, 7.46.

\section{$3 \beta$-(o-Methylbenzoyloxy)pregna-4,16-diene-6,20-dione (10c)}

White powder. Yield $62 \%$. M.p. $182-184^{\circ} \mathrm{C}$. $\mathrm{R}_{\mathrm{f}} 0.39$ (petroleum ether/ethyl acetate, 3:1, v/v). ${ }^{1} \mathrm{H} \mathrm{NMR}\left(\mathrm{CDCl}_{3}\right)$ : 0.94 (s, 3 H, H-C(18)); 1.08 (s, 3 H, H-C(19)); 2.28 (s, 3 $\mathrm{H}, \mathrm{H}-\mathrm{C}(21)) ; 2.60$ (s, $3 \mathrm{H}, \mathrm{Me}-\mathrm{Ph}) ; 5.56$ (m, $1 \mathrm{H}, \mathrm{H}-\mathrm{C}$ (3)); $6.24(\mathrm{~m}, 1 \mathrm{H}, \mathrm{H}-\mathrm{C}(4))$; $6.72(\mathrm{t}, 1 \mathrm{H}, J=1.8 \mathrm{~Hz}, \mathrm{H}-\mathrm{C}$ (16)); 7.23 (m, $2 \mathrm{H}, \mathrm{H}-\mathrm{Ph}) ; 7.40$ (m, $1 \mathrm{H}, \mathrm{H}-\mathrm{Ph}) ; 7.93$ (m, $1 \mathrm{H}, \mathrm{H}-\mathrm{Ph}) .{ }^{13} \mathrm{C} \mathrm{NMR}\left(\mathrm{CDCl}_{3}\right): 15.8(\mathrm{C}(18)) ; 19.7$ (C (19)); 27.1 (C(21)); $69.6(\mathrm{C}(3)) ; 132.1(\mathrm{C}(4)) ; 143.9(\mathrm{C}$ (16)); $148.0(\mathrm{C}(5)) ; 154.9(\mathrm{C}(17)) ; 168.4$ (ester $\mathrm{C}=\mathrm{O})$; 196.7 (C(20)); 201.8 (C(6)). IR (KBr): 3070, 2946, 1688. LC-MS: 469.4 $\left(\mathrm{M}^{+}+\mathrm{Na}\right.$, retention time $\left.2.28 \mathrm{~min}\right)$. Anal. Calcd. for $\mathrm{C}_{29} \mathrm{H}_{34} \mathrm{O}_{4}$ : C, 78.00; H, 7.67; Found: C, 77.81; $\mathrm{H}, 7.63$.

\section{3ß-( $m$-Methylbenzoyloxy)pregna-4,16-diene-6,20-dione (10d)}

White powder. Yield $69 \%$. M.p. $138-140^{\circ} \mathrm{C} . \mathrm{R}_{\mathrm{f}} 0.42$ (petroleum ether/ethyl acetate, 3:1, v/v). ${ }^{1} \mathrm{H} \mathrm{NMR}\left(\mathrm{CDCl}_{3}\right)$ : 0.95 (s, 3 H, H-C(18)); 1.11 (s, 3 H, H-C(19)); 2.27 (s, 3 $\mathrm{H}, \mathrm{H}-\mathrm{C}(21)) ; 5.60$ (m, $1 \mathrm{H}, \mathrm{H}-\mathrm{C}(3)) ; 6.24$ (m, $1 \mathrm{H}, \mathrm{H}-\mathrm{C}$ (4)); $6.71(\mathrm{t}, 1 \mathrm{H}, J=1.8 \mathrm{~Hz}, \mathrm{H}-\mathrm{C}(16))$; 7.32 (m, $2 \mathrm{H}, \mathrm{H}-$ $\mathrm{Ph}) ; 7.44(\mathrm{~m}, 1 \mathrm{H}, \mathrm{H}-\mathrm{Ph}) ; 7.84(\mathrm{~m}, 1 \mathrm{H}, \mathrm{H}-\mathrm{Ph}) .{ }^{13} \mathrm{C}$ NMR ( $\left.\mathrm{CDCl}_{3}\right)$ : 15.8 (C(18)); 19.7 (C(19)); 27.1 (C(21)); 69.8 (C(3)); $138.0(\mathrm{C}(4)) ; 143.9$ (C(16)); 148.0 (C(5)); 154.9 (C(17)); 166.4 (ester $\mathrm{C}=\mathrm{O}) ; 196.6(\mathrm{C}(20)) ; 201.9$ (C(6)). IR (KBr): 3060, 2945, 1688, 1589, 1457. LC-MS: 469.4 $\left(\mathrm{M}^{+}+\mathrm{Na}\right.$, retention time $\left.2.29 \mathrm{~min}\right)$. Anal. Calcd. for $\mathrm{C}_{29} \mathrm{H}_{34} \mathrm{O}_{4}$ : C, 78.00; $\mathrm{H}, 7.67$; Found: C, 78.18; $\mathrm{H}$, 7.71 .

\section{3 $\beta$-( $p$-Nitrobenzoyloxy)pregna-4,16-diene-6,20-dione (10e)}

Pale yellow powder. Yield $46 \%$. M.p. $174-176^{\circ} \mathrm{C} . \mathrm{R}_{\mathrm{f}} 0.36$ (petroleum ether/ethyl acetate, 3:1, v/v). ${ }^{1} \mathrm{H}$ NMR $\left(\mathrm{CDCl}_{3}\right): 0.95$ (s, $\left.3 \mathrm{H}, \mathrm{H}-\mathrm{C}(18)\right) ; 1.12$ (s, $\left.3 \mathrm{H}, \mathrm{H}-\mathrm{C}(19)\right)$; 2.28 (s, 3 H, H-C(21)); 5.62 (m, 1 H, H-C(3)); 6.21 (m, 1 $\mathrm{H}, \mathrm{H}-\mathrm{C}(4)) ; 6.72(\mathrm{t}, 1 \mathrm{H}, J=1.8 \mathrm{~Hz}, \mathrm{H}-\mathrm{C}(16)) ; 8.23(\mathrm{~m}$, $2 \mathrm{H}, \mathrm{H}-\mathrm{Ph}) ; 8.28$ (m, $2 \mathrm{H}, \mathrm{H}-\mathrm{Ph}) .{ }^{13} \mathrm{C} \mathrm{NMR}\left(\mathrm{CDCl}_{3}\right)$ : 15.8 (C(18)); 19.6 (C(19)); $27.1(\mathrm{C}(21)) ; 71.0$ (C(3)); 130.8 (C(4)); 135.4 (C(16)); 148.6 (C(5)); 154.9 (C(17)); 164.3 (ester $\mathrm{C}=\mathrm{O}$ ); $196.6(\mathrm{C}(20)) ; 201.8(\mathrm{C}(6))$. IR (KBr): 3062, 2946, 1688, 1528, 1456. LC-MS: $500.3\left(\mathrm{M}^{+}\right.$ $+\mathrm{Na}$, retention time $2.17 \mathrm{~min})$. Anal. Calcd. for $\mathrm{C}_{28} \mathrm{H}_{31} \mathrm{NO}_{6}$ : C, 70.42; H, 6.54; N, 2.93; Found: C, 70.57; $\mathrm{H}, 6.52 ; \mathrm{N}, 2.87$.

3 $\beta$-(o-Nitrobenzoyloxy)pregna-4,16-diene-6,20-dione (10f) Pale yellow powder. Yield 55\%. M.p. $158-161^{\circ} \mathrm{C}$. $\mathrm{R}_{\mathrm{f}} 0.29$ (petroleum ether/ethyl acetate, 3:1, v/v). ${ }^{1} \mathrm{H}$ NMR $\left(\mathrm{CDCl}_{3}\right): 0.92$ (s, $\left.3 \mathrm{H}, \mathrm{H}-\mathrm{C}(18)\right)$; 1.05 (s, $\left.3 \mathrm{H}, \mathrm{H}-\mathrm{C}(19)\right)$; 
2.27 (s, $3 \mathrm{H}, \mathrm{H}-\mathrm{C}(21)) ; 5.60$ (m, $1 \mathrm{H}, \mathrm{H}-\mathrm{C}(3)) ; 6.17$ (m, 1 $\mathrm{H}, \mathrm{H}-\mathrm{C}(4))$; 6.70 (t, $1 \mathrm{H}, J=1.8 \mathrm{~Hz}, \mathrm{H}-\mathrm{C}(16)) ; 7.63-7.68$ (m, $2 \mathrm{H}, \mathrm{H}-\mathrm{Ph}) ; 7.72$ (m, $1 \mathrm{H}, \mathrm{H}-\mathrm{Ph}) ; 7.95(\mathrm{~m}, 1 \mathrm{H}, \mathrm{H}-$ $\mathrm{Ph}) .{ }^{13} \mathrm{C}$ NMR $\left(\mathrm{CDCl}_{3}\right): 15.8$ (C(18)); 19.6 (C(19)); 27.1 (C(21)); 71.5 (C(3)); $133.0(\mathrm{C}(4)) ; 143.8$ (C(16)); 148.6 (C (5)); 154.9 (C(17)); 165.1 (ester $\mathrm{C}=\mathrm{O}$ ); $196.6(\mathrm{C}(20))$; 201.6 (C(6)). IR (KBr): 3033, 2945, 1732, 1687, 1656, 1628, 1587, 1540, 1456. LC-MS: $500.3\left(\mathrm{M}^{+}+\mathrm{Na}\right.$, retention time $2.11 \mathrm{~min})$. Anal. Calcd. for $\mathrm{C}_{28} \mathrm{H}_{31} \mathrm{NO}_{6}$ : C, 70.42; H, 6.54; N, 2.93; Found: C, 70.25; H, 6.49; N, 2.90 .

\section{3ß-( $m$-Nitrobenzoyloxy)pregna-4,16-diene-6,20-dione}

(10g)

Pale yellow powder. Yield $47 \%$. M.p. $176-178^{\circ} \mathrm{C} . \mathrm{R}_{\mathrm{f}} 0.38$ (petroleum ether/ethyl acetate, 3:1, v/v). ${ }^{1} \mathrm{H}$ NMR

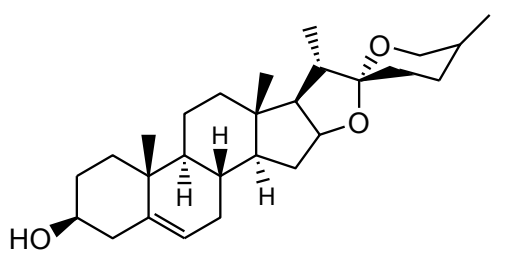

3

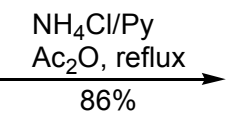<smiles>CC(=O)OCC(C)CCC1=C(C)[C@@H]2O[C@@H]3C[C@@H]4[C@@H]5CC=C6C[C@@H](OC(C)=O)CC[C@]6(C)[C@H]5CC[C@]4(C)[C@H]3[C@]2(C)CC1</smiles>

4

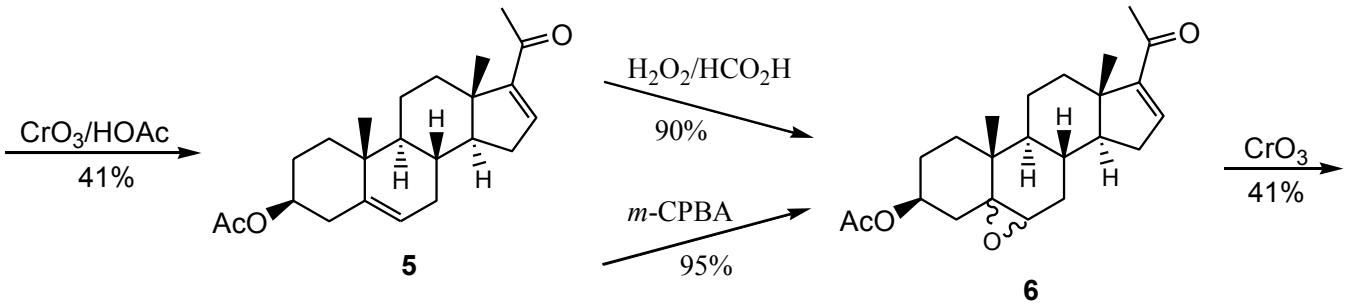

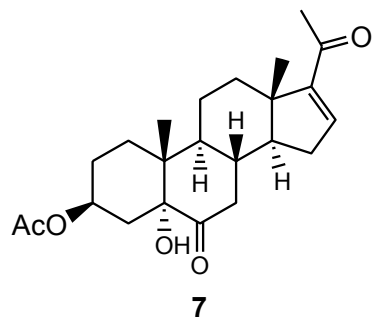

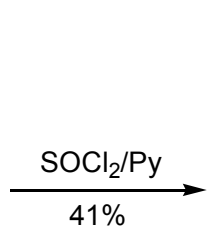<smiles>CC(=O)O[C@H]1C=C2C(=O)C[C@H]3[C@@H]4CC=C(C(C)=O)[C@@]4(C)CC[C@H]3[C@@]2(C)CC1</smiles><smiles>CC(=O)C1=CC[C@@H]2[C@@H]1CC[C@H]1[C@H]2CC(=O)C2=C[C@@H](O)CC[C@@]21C</smiles>

$\mathrm{ArCO}_{2} \mathrm{H}$ $\stackrel{\text { DCC, DMAP }}{\longrightarrow}$<smiles>CC(=O)O[C@H]1C=C2C(=O)C[C@H]3[C@H]4CC=C(C(C)=O)[C@@]4(C)CC[C@H]3[C@]2(C)CC1</smiles>
$\mathrm{Ar}=$<smiles>COc1ccc(C)cc1</smiles>

10a: $71 \%$<smiles>Cc1ccccc1[N+](=O)[O-]</smiles>
10f: $55 \%$<smiles>COc1ccccc1C</smiles>

10b: $45 \%$<smiles>Cc1cccc([N+](=O)[O-])c1</smiles>

10g: $47 \%$<smiles>Cc1ccccc1N(C)C</smiles>

10c: $62 \%$<smiles>Cc1cc([N+](=O)[O-])cc([N+](=O)[O-])c1</smiles>

10h: $45 \%$<smiles>Cc1cccc(C)c1</smiles>

10d: $69 \%$<smiles>Cc1ccccc1Cl</smiles>

10i: $64 \%$<smiles>Cc1ccc([N+](=O)[O-])cc1</smiles>

10e: $46 \%$<smiles>Cc1ccco1</smiles>

10j: $77 \%$

Figure 2 Synthetic route of target steroids 10a-j. 
Table 1 Characteristic chemical shifts in ${ }^{1} \mathrm{H}$ NMR spectra of steroids $10 \mathrm{a}-\mathrm{j}$

\begin{tabular}{llllllll}
\hline & \multicolumn{7}{c}{$\boldsymbol{\delta}$ (ppm) } \\
\cline { 2 - 8 } No. & $\mathbf{H - C}$ & $\mathbf{H - C}$ & $\mathbf{H - C}$ & $\mathbf{H}-\mathbf{C}$ & $\mathbf{H}-\mathbf{C}$ & $\mathbf{H}-\mathbf{C}$ & $\mathbf{H}-\mathbf{A r}$ \\
& $\mathbf{( 1 8 )}$ & $\mathbf{( 1 9 )}$ & $\mathbf{( 2 1 )}$ & $\mathbf{( 3 )}$ & $\mathbf{( 4 )}$ & $\mathbf{( 1 6 )}$ & \\
\hline $\mathbf{1 0 a}$ & 0.94 & 1.10 & 2.28 & 5.56 & 6.23 & 6.71 & $6.93,8.02$ \\
$\mathbf{1 0 b}$ & 0.93 & 1.07 & 2.27 & 5.56 & 6.23 & 6.73 & $6.96,7.46,7.80$ \\
$\mathbf{1 0 c}$ & 0.94 & 1.08 & 2.28 & 5.56 & 6.24 & 6.72 & $7.23,7.40,7.93$ \\
$\mathbf{1 0 d}$ & 0.95 & 1.11 & 2.27 & 5.60 & 6.24 & 6.71 & $7.30-7.38,7.84-$ \\
& & & & & & & 7.87 \\
$\mathbf{1 0 0}$ & 0.95 & 1.12 & 2.28 & 5.62 & 6.21 & 6.72 & $8.22-8.24,8.28-$ \\
& & & & & & & 8.31 \\
$\mathbf{1 0 f}$ & 0.92 & 1.05 & 2.27 & 5.60 & 6.17 & 6.70 & $7.63-7.68,7.72$, \\
& & & & & & & 7.95 \\
$\mathbf{1 0 g}$ & 0.94 & 1.12 & 2.27 & 5.63 & 6.20 & 6.71 & $7.66,8.36-8.43$, \\
& & & & & & & 8.86 \\
$\mathbf{1 0 h}$ & 0.95 & 1.15 & 2.29 & 5.70 & 6.20 & 6.72 & $9.16,9.24$ \\
$\mathbf{1 0 i}$ & 0.95 & 1.11 & 2.27 & 5.59 & 6.24 & 6.71 & $7.32,7.44,7.86$ \\
$\mathbf{1 0 j}$ & 0.94 & 1.09 & 2.28 & 5.57 & 6.20 & 6.52 & $6.71,7.20,7.59$ \\
\hline
\end{tabular}

$\left(\mathrm{CDCl}_{3}\right): 0.94(\mathrm{~s}, 3 \mathrm{H}, \mathrm{H}-\mathrm{C}(18)) ; 1.12$ (s, $\left.3 \mathrm{H}, \mathrm{H}-\mathrm{C}(19)\right)$; 2.27 (s, $3 \mathrm{H}, \mathrm{H}-\mathrm{C}(21))$; 5.63 (m, $1 \mathrm{H}, \mathrm{H}-\mathrm{C}(3))$; 6.20 (m, 1 $\mathrm{H}, \mathrm{H}-\mathrm{C}(4)) ; 6.71$ (t, J = $1.2 \mathrm{~Hz}, \mathrm{H}-\mathrm{C}(16)) ; 7.66(\mathrm{~m}, 1 \mathrm{H}$, $\mathrm{H}-\mathrm{Ph}$ ); 8.36-8.43 (m, $2 \mathrm{H}, \mathrm{H}-\mathrm{Ph}) ; 8.86$ (m, $1 \mathrm{H}, \mathrm{H}-\mathrm{Ph})$. ${ }^{13} \mathrm{C} \mathrm{NMR}\left(\mathrm{CDCl}_{3}\right)$ : $15.8(\mathrm{C}(18)) ; 19.6(\mathrm{C}(19)) ; 27.1(\mathrm{C}$ (21)); 71.0 (C(3)); $135.4(\mathrm{C}(4)) ; 143.8(\mathrm{C}(16)) ; 148.6(\mathrm{C}$ (5)); $154.9(C(17))$; 164.1 (ester $C=0) ; 196.6(C(20))$; 201.7 (C(6)). IR (KBr): 3040, 2945, 1689, 1526, 1456. LC-MS: $500.3\left(\mathrm{M}^{+}+\mathrm{Na}\right.$, retention time $\left.2.17 \mathrm{~min}\right)$. Anal. Calcd. for $\mathrm{C}_{28} \mathrm{H}_{31} \mathrm{NO}_{6}$ : C, 70.42; H, 6.54; N, 2.93; Found: C, 70.27; H, 6.58; N, 2.88 .

\section{3 $\beta$-(3,5-Dinitrobenzoyloxy)pregna-4,16-diene-6,20-dione (10h)}

Pale yellow powder. Yield $45 \%$. M.p. $168-170^{\circ} \mathrm{C} . \mathrm{R}_{\mathrm{f}} 0.59$ (petroleum ether/ethyl acetate, 2:1, v/v). ${ }^{1} \mathrm{H}$ NMR $\left(\mathrm{CDCl}_{3}\right): 0.95$ (s, $\left.3 \mathrm{H}, \mathrm{H}-\mathrm{C}(18)\right) ; 1.15$ (s, $\left.3 \mathrm{H}, \mathrm{H}-\mathrm{C}(19)\right)$; 2.29 (s, $3 \mathrm{H}, \mathrm{H}-\mathrm{C}(21)) ; 5.70$ (m, $1 \mathrm{H}, \mathrm{H}-\mathrm{C}(3))$; 6.20 (m, 1 $\mathrm{H}, \mathrm{H}-\mathrm{C}(4)) ; 6.72(\mathrm{t}, 1 \mathrm{H}, J=1.4 \mathrm{~Hz}, \mathrm{H}-\mathrm{C}(16)) ; 9.16(\mathrm{~m}$, $2 \mathrm{H}, \mathrm{H}-\mathrm{Ph}) ; 9.24(\mathrm{~m}, 1 \mathrm{H}, \mathrm{H}-\mathrm{Ph}) .{ }^{13} \mathrm{C} \mathrm{NMR}\left(\mathrm{CDCl}_{3}\right)$ : 15.8 (C(18)); 19.5 (C(19)); $27.1(\mathrm{C}(21)) ; 72.2$ (C(3)); 133.8 (C(4)); 143.8 (C(16)); 148.6 (C(5)); 154.8 (C(17)); 162.2 (ester $\mathrm{C}=\mathrm{O})$; $196.6(\mathrm{C}(20)) ; 201.6(\mathrm{C}(6))$. IR (KBr): 3037, 2946, 1689, 1543, 1457. LC-MS: $500.3\left(\mathrm{M}^{+}\right.$ $+\mathrm{Na}$, retention time $3.14 \mathrm{~min})$. Anal. Calcd. for $\mathrm{C}_{28} \mathrm{H}_{30} \mathrm{~N}_{2} \mathrm{O}_{8}$ : C, 64.36; H, 5.79; N, 5.36; Found: C, 64.22; H, 5.75; N, 5.19.

\section{$3 \beta$-(o-Chlorobenzoyloxy)pregna-4,16-diene-6,20-dione (10i)}

White powder. Yield 64\%. M.p. $192-194^{\circ} \mathrm{C} . \mathrm{R}_{\mathrm{f}} 0.32$ (petroleum ether/ethyl acetate, 3:1, v/v). ${ }^{1} \mathrm{H} \mathrm{NMR}\left(\mathrm{CDCl}_{3}\right)$ : 0.95 (s, 3 H, H-C(18)); 1.11 (s, 3 H, H-C(19)); 2.27 (s, 3
$\mathrm{H}, \mathrm{H}-\mathrm{C}(21)) ; 5.59$ (m, $1 \mathrm{H}, \mathrm{H}-\mathrm{C}(3)) ; 6.24(\mathrm{~m}, 1 \mathrm{H}, \mathrm{H}-\mathrm{C}$ (4)); 6.71 (t, $1 \mathrm{H}, J=1.8 \mathrm{~Hz}, \mathrm{H}-\mathrm{C}(16)) ; 7.32(\mathrm{~m}, 1 \mathrm{H}, \mathrm{H}-$ $\mathrm{Ph}) ; 7.36$ (m, $2 \mathrm{H}, \mathrm{H}-\mathrm{Ph}) ; 7.87$ (m, $1 \mathrm{H}, \mathrm{H}-\mathrm{Ph}) .{ }^{13} \mathrm{C}$ NMR ( $\left.\mathrm{CDCl}_{3}\right)$ : 15.8 (C(18)); 19.6 (C(19)); 27.1 (C(21)); 70.5 (C(3)); 133.9 (C(4)); 143.8 (C(16)); 148.2 (C(5)); 154.9 (C(17)); 165.2 (ester $\mathrm{C}=\mathrm{O}) ; 196.6(\mathrm{C}(20)) ; 201.7$ (C(6)). IR (KBr): 3040, 2946, 1688, 1522, 1456. LC-MS: $490.2\left(\mathrm{M}^{+}+\mathrm{Na}\right.$, retention time $\left.2.23 \mathrm{~min}\right)$. Anal. Calcd. for $\mathrm{C}_{28} \mathrm{H}_{31} \mathrm{ClO}_{4}$ : C, 72.01; $\mathrm{H}, 6.69$; Found: C, 71.83; $\mathrm{H}$, 6.65 .

\section{3ß-(2-Furoyloxy)pregna-4,16-diene-6,20-dione (10j)}

White powder. Yield $77 \%$. M.p. $180-182^{\circ} \mathrm{C}$. $\mathrm{R}_{\mathrm{f}} 0.40$ (petroleum ether/ethyl acetate, 2:1, v/v). ${ }^{1} \mathrm{H} \mathrm{NMR}\left(\mathrm{CDCl}_{3}\right)$ : 0.94 (s, 3 H, H-C(18)); 1.09 (s, 3 H, H-C(19)); 2.28 (s, 3 $\mathrm{H}, \mathrm{H}-\mathrm{C}(21)$ ); 5.57 (m, $1 \mathrm{H}, \mathrm{H}-\mathrm{C}(3)) ; 6.20$ (m, $1 \mathrm{H}, \mathrm{H}-\mathrm{C}$ (4)); 6.52 (t, $1 \mathrm{H}, J=1.8 \mathrm{~Hz}, \mathrm{H}-\mathrm{C}(16)) ; 6.71$ (m, $1 \mathrm{H}, \mathrm{H}-$ Ar); $7.20(\mathrm{~m}, 1 \mathrm{H}, \mathrm{H}-\mathrm{Ar}) ; 7.59$ (m, $1 \mathrm{H}, \mathrm{H}-\mathrm{Ar}) .{ }^{13} \mathrm{C}$ NMR ( $\left.\mathrm{CDCl}_{3}\right)$ : 15.8 (C(18)); 19.6 (C(19)); 27.1 (C(21)); 69.9 (C(3)); 128.7 (C(4)); 143.8 (C(16)); 148.1 (C(5)); 154.8 (C(17)); 158.3 (ester $\mathrm{C}=\mathrm{O}) ; 196.6(\mathrm{C}(20)) ; 201.8$ (C(6)). IR (KBr): 3042, 2946, 1720, 1689, 1657, 1580, 1523, 1457. LC-MS: $445.2\left(\mathrm{M}^{+}+\mathrm{Na}\right.$, retention time 2.06 min). Anal. Calcd. for $\mathrm{C}_{26} \mathrm{H}_{30} \mathrm{O}_{5}: \mathrm{C}, 73.91 ; \mathrm{H}, 7.16$; Found: C, 73.73; H, 7.21.

\section{Results and Discussion}

The synthetic route of the target steroids $3 \beta$-benzoyloxypregna-4,16-diene-6,20-dione (10a-i) and 3 $\beta$-furoyloxypregna-4,16-diene-6,20-dione (10j) was showed in Figure 2. At first the starting material diosgenin was transformed by acetylation and oxidation to $3 \beta$-acetoxy5,16-pregnadiene-20-one (16-DPA, 5). The transformation could be achieved stepwise or in a one-pot reaction [32]. We adopted the former in consideration that the purification of the product was easier to yield 16-DPA of higher purity. Epoxidation of 16-DPA to form steroid 6 was conducted by using $30 \% \mathrm{H}_{2} \mathrm{O}_{2} / \mathrm{HCO}_{2} \mathrm{H}$ [33] or $m$-chloroperoxybenzoic acid (m-CPBA) [13] oxidation system in $90 \%$ or $95 \%$ yield.

Conversion of compound $\mathbf{6}$ to 7 was carried out in a similar way as reported in literature [13] and the yield of steroid 7 was improved from $81 \%$ to $84 \%$. Dehydration of 7 with thionyl chloride in pyridine resulted compound 8. We found that the quality of the solvent pyridine had an effect on the yield of the steroid $\mathbf{8}$. When dried pyridine (refluxed in the presence of potassium hydroxide for $2 \mathrm{~h}$ before distillation) was used instead of pyridine of analytically pure grade without treatment, the yield of $\mathbf{8}$ was improved to $73 \%$ from the literature [13] value $66 \%$. Compound 8 was hydrolyzed with aqueous sodium hydroxide in methanol to afford $3 \beta$-hydroxypregna-4,16-diene-6,20-dione (9). The procedure for workup was improved by using extraction (with 
Table 2 Crystal data and experimental details for compound 10a

\begin{tabular}{ll}
\hline Empirical formula & C29 H34 O5 \\
Formula weight & 462.56 \\
Temperature & $296(2) \mathrm{K}$ \\
Wavelength & $0.71073 \AA$ \\
Crystal system, space group & Monoclinic, P2(1) \\
Unit cell dimensions & $a=10.9972(10) \AA \alpha=90^{\circ}$ \\
& $b=7.4244(7) \AA \beta=108.7520(10)^{\circ}$ \\
& $c=15.8025(15) \AA \gamma=90^{\circ}$ \\
Volume & $1221.7(2) \AA^{3}$ \\
Z, Calculated density & $2,1.257 \mathrm{Mg} / \mathrm{m}^{3}$ \\
Absorption coefficient & $0.085 \mathrm{~mm}^{-1}$ \\
F(000) & 496 \\
Crystal size & $0.12 \times 0.10 \times 0.08 \mathrm{~mm}$ \\
$\theta$ range for data collection & $2.72<\theta<24.99^{\circ}$ \\
Reflections collected/unique & $10638 / 4269[R$ (int) $=0.0219]$ \\
Absorption correction & Semi-empirical from equivalents \\
Max. and min. transmission & 0.9933 and 0.9899 \\
Refinement method & Full-matrix least-squares on $F^{2}$ \\
Data/restraints/parameters & $4269 / 1 / 311$ \\
Goodness-of-fit on $F^{2}$ & 0.999 \\
Final $R$ indices [I $>$ 2 $\sigma$ (I)] & $R 1=0.0377, w R 2=0.0884$ \\
$R$ indices (all data) & $R 1=0.0492, w R 2=0.0958$ \\
Absolute structure parameter & $0.1(11)$ \\
Largest diff. peak and hole & 0.167 and -0.140 e $\AA^{-3}$ \\
\hline
\end{tabular}

ethyl acetate) and concentration replacing filtration and subsequent recrystallization and pure product 9 was obtained in $74 \%$ yield, significantly higher than the literature [13] value $60 \%$.

The target steroids $3 \beta$-benzoyloxypregna-4,16-diene6,20-dione (10a-i) and $3 \beta$ - furoyloxypregna-4,16-diene6,20-dione (10j) were synthesized through Steglich reaction from 9, substituted benzoic acid or furoic acid, and DCC catalyzed by DMAP in fair to good yields (45\%-
77\%), depending upon the structure of the aryl acids. It seems that the steric hindrance from the aryl acid structure leads to decrease in the yield. Therefore the highest yield of the Steglich reaction came from furoic acid (77\%) while the reactions with dinitrobenzoic acid or nitrobenzoic acid gave the lowest yield.

The chemical structures of steroids $10 \mathbf{a}-\mathbf{j}$ were fully characterized by elemental analysis, ${ }^{1} \mathrm{H}$ NMR, ${ }^{13} \mathrm{C}$ NMR, IR and mass spectrum and melting point measurements. Characteristic chemical shifts in ${ }^{1} \mathrm{H}$ NMR spectra of steroids 10a-j were summarized in Table 1 . All the chemical shifts of protons in methyl groups, $\mathrm{OCH}$ moieties, $\mathrm{C}=\mathrm{CH}$ moieties, and aryl rings were in the reasonable range and the spectra data were in good accordance with the structures.

Single crystals of 10a suitable for X-ray crystal diffraction measurement were obtained by slow evaporation of a solution of 10a in 1:1 petroleum ether and ethyl acetate. X-Ray data for crystals of 10a were collected by graphite-monochromatized Mo $K_{\alpha}$ radiation at $293 \mathrm{~K}$ and the crystal data and experimental details for compound 10a were summarized in Table 2. The structure was solved by direct methods and refined by full-matrix least-squares with anisotropic temperature factors for the non-hydrogen atoms. The hydrogen atoms bonded to the carbon atoms were assigned based on the expected bonding geometry. The hydrogen atoms were refined isotropically in the final least-squares cycles.

The crystal structure and molecular packing of steroid 10a were shown in Figure 3 and Figure 4 respectively. It can be seen that the molecule consists of three sixmembered rings ( $\mathrm{A}, \mathrm{B}$, and $\mathrm{C}$ ring) and one five-membered ring ( $\mathrm{D}$ ring), all trans fused. The six-membered rings $\mathrm{A}, \mathrm{B}$, and $\mathrm{C}$ occur in a half chair, distorted chair, and distorted chair conformation, respectively. Ring D adopts a distorted envelope conformation. The molecules in the crystal were packed face-to-face at the

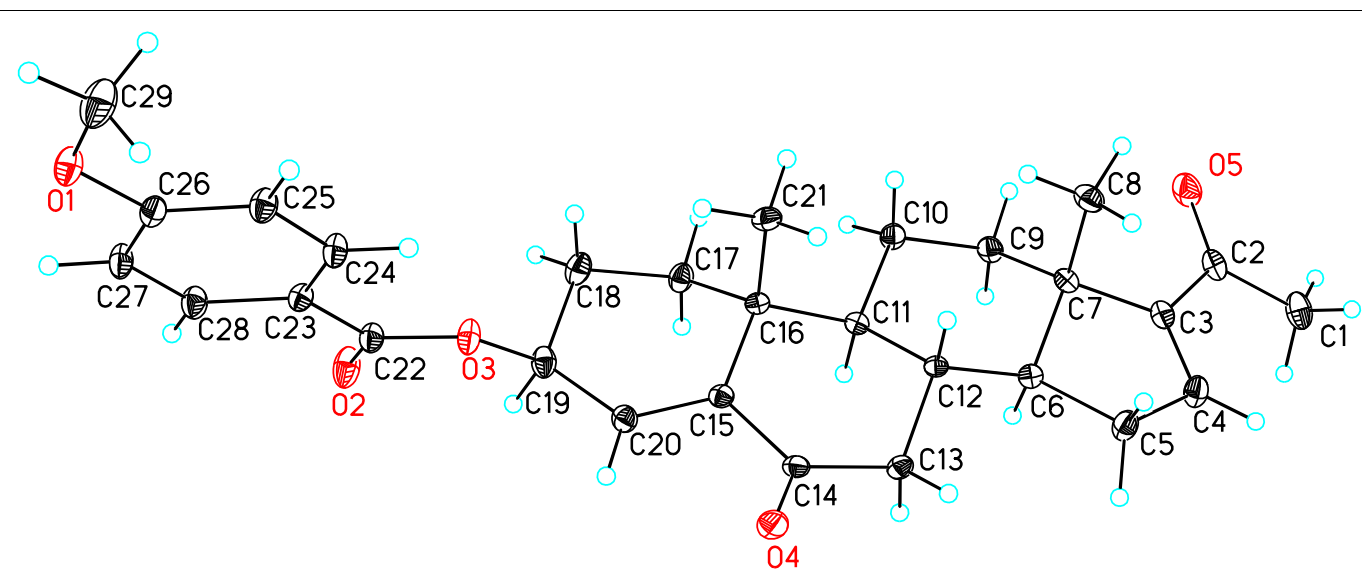

Figure 3 Crystal structure of compound 10a. 


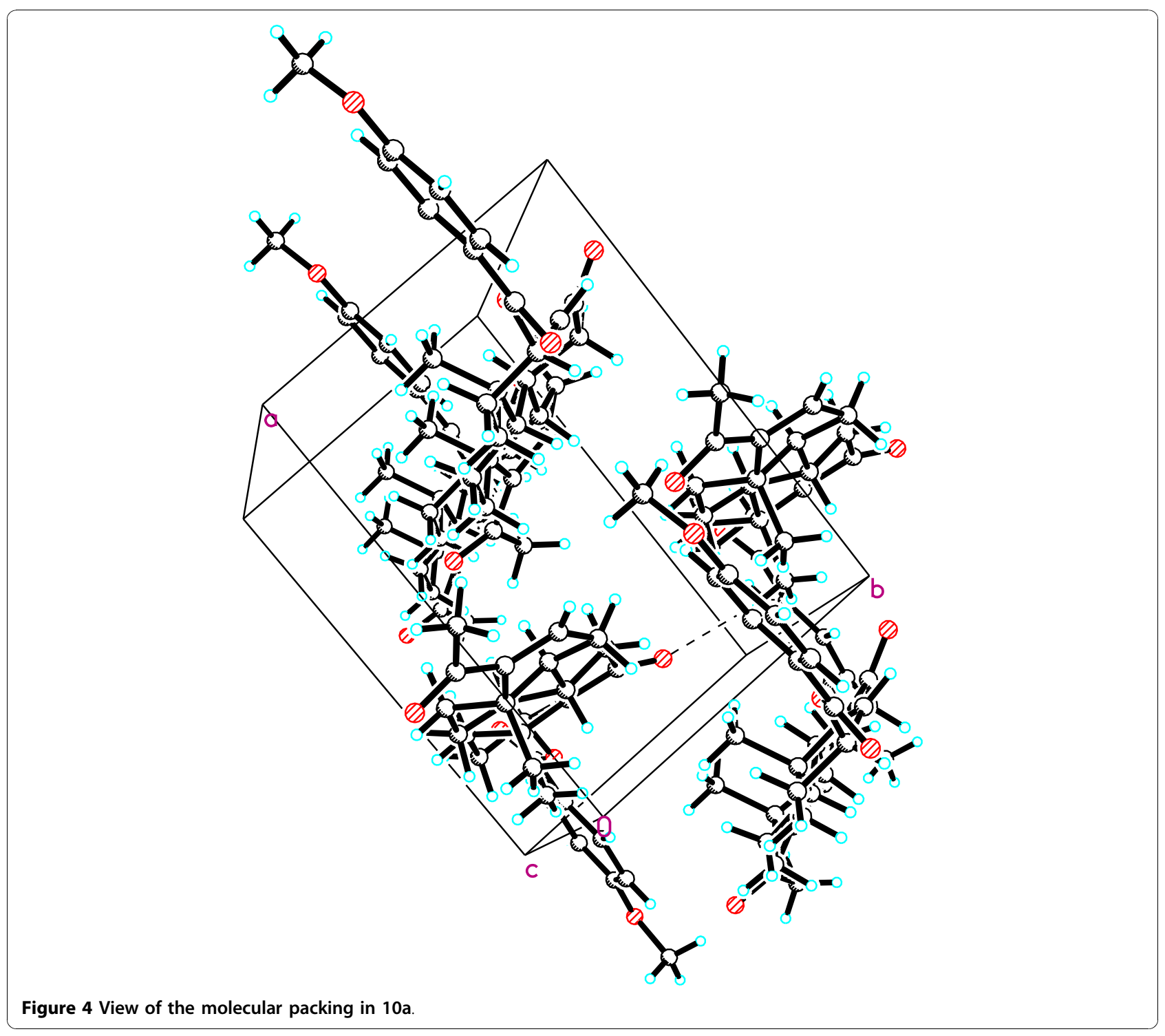

normal van der Waals distances, similar to the crystal structure of $3 \beta$-( $p$-fluorobenzoyloxy)-4,16-pregnadiene6,20-dione [34]. CCDC-787721 contains the supplementary crystallographic data for this paper. These data can be obtained free of charge via http://www.ccdc.cam.ac. uk/data_request/cif.

\section{Conclusions}

In summary, ten new aromatic esters based on 4,16-pregnadiene-6,20-dione skeleton, namely $3 \beta$-benzoyloxy-4,16pregnadiene-6,20-dione and $3 \beta$-furoyloxy-4,16-pregnadiene-6,20- dione were synthesized starting from diosgenin. The structures of the steroids were characterized by elemental analysis, ${ }^{1} \mathrm{H}$ NMR, ${ }^{13} \mathrm{C}$ NMR, IR and mass spectrum. Single crystal X-ray diffraction measurement on one of the new compounds, $3 \beta$-( $p$-methoxybenzoyloxy)-4,16- pregnadiene-6,20-dione revealed that the A, B, C, and D ring adopted half chair, distorted chair, distorted chair, and distorted envelope conformation, respectively. The molecules in the crystal were packed face-to-face at the normal van der Waals distances. These new steroids may show high antiandrogenic activity and serve as good inhibitors for the $5 \alpha$-reductase enzyme. Investigation of the inhibitory activity of the new steroids for the $5 \alpha$-reductase enzyme will be conducted in the near future.

\section{Acknowledgements}

The authors acknowledge the financial support of the project by Shanghai Natural Science Foundation (No. 06ZR14001).

\section{Authors' contributions}

$J \mathrm{~L}$ carried out the synthetic experiments and drafted the manuscript. $\mathrm{HL}$ completed the molecular design, the arrangement of the work and 
modification of the manuscript. YL participated in the separation and purification of compounds, acquisition of data, and collection of literature and helped to draft the manuscript. All authors read and approved the final manuscript.

\section{Competing interests}

The authors declare that they have no competing interests.

Received: 21 October 2010 Accepted: 8 December 2010 Published: 8 December 2010

\section{References}

1. Yarrow JF, McCoy SC, Borst SE: Tissue selectivity and potential clinical applications of trenbolone (17 $\beta$-hydroxyestra-4,9,11-trien-3-one): a potent anabolic steroid with reduced androgenic and estrogenic activity. Steroids 2010, 75:377-389.

2. Morioka M, Kamizono A, Takikawa H, Mori A, Ueno H, Kadowaki S, Nakao Y, Kato K, Umezawa K: Design, synthesis, and biological evaluation of novel estradiol-bisphosphonate conjugates as bone-specific estrogens. Bioorg Med Chem 2010, 18:1143-1148.

3. Reyes-Moreno M, Ruiz-García JA, Ibarra-Reyes Y, Fuente-Hernández A, VélezCastro H, Hernández-Balmaseda I, Martínez-Hormaza I, Rodeiro-Guerra I, Ramírez JS, Reyes SM, Montiel-Smith S: Synthesis and anabolic/androgenic evaluation of novel 9a-fluorosteroids. Eur J Med Chem 2009, 44:4567-4571.

4. Bunyathaworn P, Boonananwong S, Kongkathip B, Kongkathip N: Further study on synthesis and evaluation of 3,16,20-polyoxygenated steroids of marine origin and their analogs as potent cytotoxic agents. Steroids 2010, 75:432-444.

5. Kim T-K, Chen J, Li W, Zjawiony J, Miller D, Janjetovic Z, Tuckey RC, Slominski A: A new steroidal 5,7-diene derivative, 3ß-hydroxyandrosta5,7-diene-17 $\beta$ - carboxylic acid, shows potent anti-proliferative activity. Steroids 2010, 75:230-239.

6. Rasras AJM, Al-Tel TH, Al-Aboudi AF, Al-Qawasmeh RA: Synthesis and antimicrobial activity of cholic acid hydrazone analogues. Eur J Med Chem 2010, 45:2307-2313.

7. Leng TD, Zhang JX, Xie J, Zhou SJ, Huang YJ, Zhou YH, Zhu WB, Yan GM: Synthesis and anti-glioma activity of $25(\mathrm{R})$-spirostan-3 $3,5 \alpha, 6 \beta, 19$-tetrol. Steroids 2010, 75:224-229.

8. Gunasekara S, Vrielink A, Stubbs KA: Preliminary studies into the inhibition of the cholesterol a-glucosyltransferase from Helicobacter pylori using azasugars. Carbohyd Res 2010, 345:960-964.

9. Dubey S, Sharma AK, Jindal DP, Harvey A, Singh R, Bodhankar SL: Synthesis and neuromuscular blocking activity of 16-(2- and 3-pyridylmethylene) dehydroepiandrosterone derivatives. Steroids 2010, 75:323-329.

10. Bastien D, Leblanc V, Asselin É, Bérubé G: First synthesis of separable isomeric testosterone dimers showing differential activities on prostate cancer cells. Bioorg Med Chem Lett 2010, 20:2078-2081.

11. Liu J, Zhang X, Zhao M, Peng S: Synthesis, evaluation and 3D QSAR analysis of novel estradiol-RGD octapeptide conjugates with oral antiosteoporosis activity. Eur J Med Chem 2009, 44:1689-1704.

12. Weng Y, Xiang L, Matsuura A, Zhang Y, Huang Q, Qi J: Ganodermasides A and $B$, two novel anti-aging ergosterols from spores of a medicinal mushroom Ganoderma lucidum on yeast via UTH1 gene. Bioorg Med Chem 2010, 18:999-1002.

13. Pérez-Ornelas V, Cabeza M, Bratoeff E, Heuze I, Sánchez M, Ramírez E, Naranjo-Rodríguez E: New 5a-reductase inhibitors: in vitro and in vivo effects. Steroids 2005, 70:217-224.

14. Chapman KE, Odermatt A: Steroids: modulators of inflammation and immunity. J Steroid Biochem Mol Biol 2010, 120:67-68.

15. Iványi Z, Wölfling J, Görbe T, Szécsi M, Wittmann T, Schneider G: Synthesis of regioisomeric $17 \beta-N$-phenylpyrazolyl steroid derivatives and their inhibitory effect on 17a-hydroxylase/ $\mathrm{C}_{17,20}$-lyase. Steroids 2010 , 75:450-456.

16. Källström A-C, Salme R, Rydén L, Nordenskjöld B, Jönsson P-E, Stål O: 17ßHydroxysteroid dehydrogenase type 1 as predictor of tamoxifen response in premenopausal breast cancer. Eur J Cancer 2010, 46:892-900.

17. Cabeza M, García-Lorenzana M, Garcés M, Heuze I, Teran N, Bratoeff E: New$D$ - homoandrost-4,6-diene derivatives as potent progesterone receptor antagonist. Steroids 2010, 75:101-108.
18. Wuest F, Carlson KE, Katzenellenbogen JA: Expeditious synthesis of steroids containing a 2-methylsulfanyl-acetyl side chain as potential glucocorticoid receptor imaging agents. Steroids 2008, 73:69-76.

19. Xu J-Q, Shen $\mathrm{Q}, \mathrm{Li}$ J, Hu L-H: Dammarans from Gynostemma pentaphyllum and synthesis of their derivatives as inhibitors of protein tyrosine phosphatase 1B. Bioorg Med Chem 2010, 18:3934-3939.

20. Xiong J, Taniguchi M, Kashiwada Y, Sekiya M, Yamagishi T, Takaishi Y: Papyriferic acid derivatives as reversal agents of multidrug resistance in cancer cells. Bioorg Med Chem 2010, 18:2964-2975.

21. Bydal P, Luu-The V, Labrie F, Poirier D: Steroidal lactones as inhibitors of 17ß-hydroxysteroid dehydrogenase type 5: chemical synthesis, enzyme inhibitory activity, and assessment of estrogenic and androgenic activities. Eur J Med Chem 2009, 44:632-644.

22. Yaya AR, Touaibia M, Massarweh G, Rochon FD, Breau L: Synthesis of 17asubstituted ethynylestradiols: potential ligands for drug vectors. Steroids 2010, 75:489-498.

23. Novakova V, Zimcik P, Miletin M, Kopecky K, Ivincová J: A phthalocyaninemestranol conjugate for photodynamic therapy prepared via click chemistry. Tetrahedron Lett 2010, 51:1016-1018.

24. Oaksmith JM, Ganem B: Synthesis of a COMC-estradiol conjugate for targeted, tissue-selective cancer chemotherapy. Tetrahedron Lett 2009, 50:3497-3498.

25. Radchatawedchakoon W, Watanapokasin R, Krajarng A, Yingyongnarongkul B: Solid phase synthesis of novel asymmetric hydrophilic head cholesterol-based cationic lipids with potential DNA delivery. Bioorg Med Chem 2010, 18:330-342.

26. Köysal O: Conductivity and dielectric properties of cholesteric liquid crystal doped with single wall carbon nanotube. Synth Met 2010, 160:1097-1100.

27. Edelsztein VC, Burton G, Di Chenna PH: Self-assembly of a silylated steroid-based organogelator and its use as template for the in situ solgel polymerization of tetraethyl orthosilicate. Tetrahedron 2010, 66:2162-2167.

28. Cabeza M, Heuze I, Sánchez M, Bratoeff E, Ramírez E, Rojas A, Orozco A, Mungía A, Agustín G, Cuatepotzo L, Gonzalez C, Palma S, Padilla D, Perez V, Jimenez G: Relative binding affinity of novel steroids to androgen receptors in hamster prostate. J Enzyme Inhib Med Chem 2005, 20:357-364.

29. Cabeza M, Bratoeff E, Ramírez E, Heuze I, Recillas S, Berrios H, Cruz A, Cabrera O, Perez V: Biological activity of novel progesterone derivatives having a bulky ester side chains at C-3. Steroids 2008, 73:838-843.

30. Li H, Song Y, Peng X: Improved synthesis of mestranol and ethinyl estradiol (EE) related degradation products as authentic references. Steroids 2008, 73:488-494

31. Li H, Song Y, Ge F: 17a-Ethynyl-3-methoxyestra-1,3,5(10),9(11)-tetraen-17ol. Acta Crystallogr E 2008, 64:0783.

32. Bandhoria P, Gupta VK, Gupta DK, Jain SM, Varghese B: Crystal structure of 3ß-acetoxy-pregna-5,16-dien-20-one (16 DPA). J Chem Crystallogr 2006, 36:161-166.

33. Sun F, Lu H, Ge W: Synthesis of epoxy-progesterone derivatives and spectroscopic analysis. J Mol Sci (in Chinese) 2007, 23:209-212.

34. Soriano-García M, Valencia N, Flores E, Bratoeff E, Ramírez E, Cabeza M: Crystal structure and synthesis of $\beta$-(p-fluorobenzoyloxy)-4,16pregnadiene-6,20-dione. Anal Sci 2003, 19:x79-x80.

doi:10.1186/1752-153X-4-18

Cite this article as: $L i$ et al: Synthesis and characterization of new aromatic esters based on 4,16-pregnadiene-6,20-dione skeleton. Chemistry Central Journal 2010 4:18. 